\title{
Circuit-Specific Alterations in Hippocampal Synaptophysin Immunoreactivity Predict Spatial Learning Impairment in Aged Rats
}

\author{
Thressa D. Smith, ${ }^{1}$ Michelle M. Adams, ${ }^{1,2}$ Michela Gallagher, ${ }^{4}$ John H. Morrison, ${ }^{1,2,3}$ and Peter R. Rapp ${ }^{1,2,3}$ \\ ${ }^{1}$ Kastor Neurobiology of Aging Laboratories, ${ }^{2}$ Fishberg Research Center for Neurobiology, and ${ }^{3}$ Department of Geriatrics \\ and Adult Development, Mount Sinai School of Medicine, New York, New York 10029-6574, and ${ }^{4}$ Department of \\ Psychology, Johns Hopkins University, Baltimore, Maryland 21218-2686
}

\begin{abstract}
The present study examined the long-standing concept that changes in hippocampal circuitry contribute to age-related learning impairment. Individual differences in spatial learning were documented in young and aged Long-Evans rats by using a hippocampal-dependent version of the Morris water maze. Postmortem analysis used a confocal laser-scanning microscopy method to quantify changes in immunofluorescence staining for the presynaptic vesicle glycoprotein, synaptophysin (SYN), in the principal relays of hippocampal circuitry. Comparisons based on chronological age alone failed to reveal a reliable difference in the intensity of SYN staining in any region that was examined. In contrast, aged subjects with spatial learning deficits displayed significant reductions in SYN immunoreactivity in CA3 lacunosum-moleculare (LM) relative to either young controls or age-matched rats with preserved learning. SYN intensity values
\end{abstract}

for the latter groups were indistinguishable. In addition, individual differences in spatial learning capacity among the aged rats correlated with levels of SYN staining selectively in three regions: outer and middle portions of the dentate gyrus molecular layer and CA3-LM. The cross-sectional area of SYN labeling, by comparison, was not reliably affected in relation cognitive status. These findings are the first to demonstrate that a circuit-specific pattern of variability in the connectional organization of the hippocampus is coupled to individual differences in the cognitive outcome of normal aging. The regional specificity of these effects suggests that a decline in the fidelity of input to the hippocampus from the entorhinal cortex may play a critical role.

Key words: circuit organization; synaptophysin; hippocampus; entorhinal cortex; aging; spatial learning; Morris water maze
A substantial proportion of aged individuals exhibit learning and memory deficits that qualitatively resemble the effects of direct hippocampal damage (for review, see Gallagher and Rapp, 1997). Related alterations are observed in hippocampal neuronal activity in aged rats with spatial learning deficits, including a decline in the scope of information controlling location-specific firing, and modified place field stability (Barnes et al., 1997; Shen et al., 1997; Tanila et al., 1997a,b). Against this background recent stereological investigations indicate that the total number of dentate gyrus granule cells and pyramidal neurons in fields CA3/2 and CA1 remains stable in aged mice (Calhoun et al., 1998), rats (Rapp and Gallagher, 1996; Rasmussen et al., 1996), monkeys (Rapp, 1995; Peters et al., 1996), and humans (West, 1993). In the subset of these studies incorporating behavioral assessment, neuron number also was preserved among aged subjects that displayed robust deficits on tests of learning and memory that depend on the hippocampal formation (Rosene, 1993; Rapp, 1995; Rapp and Gallagher, 1996; Rasmussen et al., 1996). Together, these findings have prompted a consensus that hippocampal information processing can deteriorate during normal aging in the absence of significant neuronal loss (Gallagher et al., 1996; Morrison and Hof, 1997).

Studies of synaptic density suggest that hippocampal connectivity is more susceptible to aging (for review, see Coleman and Flood, 1987; deToledo-Morrell et al., 1988a; Geinisman et al., 1995). Of particular note, Geinisman et al. (1986) documented a decline in a morphologically distinct population of synapses in the dentate gyrus molecular layer that was coupled to the magnitude of age-related learning impairment. Other studies have reported changes in the relative volume of defined components of hippocampal circuitry (Coleman et al., 1987;

\footnotetext{
Received April 6, 2000; revised June 5, 2000; accepted June 6, 2000.

This work was supported by National Institutes of Health Grants AG09973 (P.R.R. and M.G.) and AG06647 (J.H.M.). We thank Robert McMahan for expert technical assistance.

Correspondence should be addressed to Dr. Peter R. Rapp, Kastor Neurobiology of Aging Laboratories, Mount Sinai School of Medicine, Box 1639, One Gustave L. Levy Place, New York, NY 10029-6574. E-mail: rapp@neuro.mssm.edu.

Copyright (C) 2000 Society for Neuroscience $0270-6474 / 00 / 206587-07 \$ 15.00 / 0$
}

Rapp et al., 1999), and, in one case, these effects were related to the severity of age-related learning impairment (Rapp et al., 1999).

Commenting on available evidence, Geinisman (1999) and others (Barnes, 1999; Nicolle et al., 1999a; Smith et al., 1999) have noted the lack of high-resolution anatomically comprehensive analyses documenting the status of hippocampal circuitry in relation to the cognitive outcome of aging. In addition, previous studies have focused primarily on synapse number and density, leaving open the potential contribution of alterations in other parameters affecting the integrity of hippocampal connectivity. The present investigation addressed these issues by using confocal laser-scanning microscopy (CLSM) to quantify immunohistochemical labeling for the presynaptic vesicle marker synaptophysin (SYN-IR). This approach allows for extensive regional sampling in large numbers of subjects and yields multiple measures of circuit integrity (Cabalka et al., 1992; Good et al., 1992; Masliah et al., 1993, 1994, 1995; Chen et al., 1995; Gazzaley et al., 1996). A central element of the experimental design was the use of a well characterized behavioral assessment that is sensitive to hippocampal disruption (Morris et al., 1982) and that reveals significant learning impairment in a substantial proportion of aged subjects (Gallagher and Rapp, 1997). By this strategy our aim was to conduct an anatomically comprehensive analysis of hippocampal circuitry in relation to individual variability in normal cognitive aging.

\section{MATERIALS AND METHODS}

Subjects. Twenty-seven young (6 months; $n=10)$ and aged (24-28 months; $n=17$ ) male Long-Evans hooded rats (Charles River Laboratories, Raleigh, NC) served as subjects. Animals were housed singly in standard cages in a climate controlled vivarium (ambient temperature, $25^{\circ} \mathrm{C}$ ) maintained on a $12 \mathrm{hr}$ light/dark cycle. Food and water were available ad libitum. Sentinel screening for a panel of viral antibodies proved negative, confirming the pathogen-free status of aged animals from this colony. Husbandry and experimental procedures followed the National Institutes of Health Guide for the Care and Use of Laboratory Animals and were approved by institutional Animal Care and Use Committees.

Behavioral testing procedures. The apparatus and protocol for evaluating spatial learning in the Morris water maze were the same as in numerous earlier experiments (Gallagher et al., 1993; Rapp and Gallagher, 1996; Rapp et al., 1999). Briefly, rats were tested on a standardized "place" 
version of the water maze task for a total of $8 \mathrm{~d}$ consecutively. Three trials were provided per day with $60 \mathrm{sec}$ intertrial intervals. The location of the hidden escape platform remained constant across trials relative to the distribution of spatial cues surrounding the apparatus. Animals entered the maze at one of four points around the perimeter of the apparatus, according to a predetermined sequence. Rats that failed to escape within $90 \mathrm{sec}$ were guided to the platform where they remained for $30 \mathrm{sec}$. The last trial on every other day was a probe test in which the escape platform was unavailable for escape for the first $30 \mathrm{sec}$. Throughout testing the search paths were monitored by a video tracking system (HVS Image Analyzing VP-112) and analyzed with custom-designed software (developed by Richard Baker at HVS Imaging, Hampton, UK). The distance between the rat and the escape platform was sampled 10 times/sec and averaged in $1 \mathrm{sec}$ bins; then two standardized behavioral measures were derived (Gallagher et al., 1993). To evaluate performance during training trials, we calculated cumulative search error as the summed $1 \mathrm{sec}$ averages of the proximity measure. At the outset of training this measure has a high value but approaches zero as the rats learn to swim directly to the platform from any start location. A learning index score of spatial bias also was calculated on the basis of data that were collected during probe testing; lower index scores reflect searching focused on the target location and indicate better learning.

Animals subsequently were tested for one session of six trials $(30 \mathrm{sec}$ intertrial interval) on a nonspatial hippocampal-independent version of the water maze. For this "cued" task the platform was visible and varied in location across trials. Previous studies indicate that performance in aged rats from the present study population is unimpaired on this nonspatial version of testing (for review, see Gallagher and Rapp, 1997).

Histological and immunohistochemical procedures. Rats were shipped to the Mount Sinai School of Medicine vivarium 7-10 d after behavioral testing. After 1-2 weeks of acclimation they were anesthetized deeply with chloral hydrate $(30 \%$, i.p.) and were perfused transcardially with ice-cold $1 \%$ paraformaldehyde in $0.1 \mathrm{M}$ PBS for $1 \mathrm{~min}$, followed by $9-14 \mathrm{~min}$ of ice-cold $4 \%$ paraformaldehyde in PBS with $0.125 \%$ glutaraldehyde at a flow rate of $65 \mathrm{ml} / \mathrm{min}$. Animals with grossly apparent pituitary hypertrophy were excluded. Brains were blocked in the coronal plane and post-fixed for $6 \mathrm{hr}$. Tissue blocks were stored in PBS with $0.1 \%$ sodium azide at $4{ }^{\circ} \mathrm{C}$. Histological sections through the rostrocaudal extent of the hippocampus were cut on vibratome at a nominal thickness of $50 \mu \mathrm{m}$. Serial sections were collected in PBS with $0.1 \%$ sodium azide and stored at $4^{\circ} \mathrm{C}$ until immunohistochemical processing (generally within 1 week).

A one-in-ten series of histological sections (500 $\mu \mathrm{m}$ spacing) was labeled with a monoclonal antibody against SYN $(0.10 \mu \mathrm{g} / \mathrm{ml}$ final antibody dilution; Boehringer Mannheim, Indianapolis, IN). According to this design an average of eight sections per brain (total 206) was included in the main analysis. An alternate series of more widely spaced sections $(1 \mathrm{~mm} \mathrm{spac}-$ ing) from the same brains was processed for the visualization of microtubule-associated protein 2 (MAP2), using a commercially available monoclonal antibody $(20 \mu \mathrm{g} / \mathrm{ml}$ final antibody dilution; Sigma, St. Louis, MO). The characteristics and specificity of immunostaining have been documented for both antibodies (Bernhardt and Matus, 1984; Wiedenmann and Franke, 1985; Gazzaley et al., 1996).

Material was coprocessed in batches of approximately three young and six aged brains (representing a wide range of spatial learning capacities) with the same reagents. This design eliminated the possibility that differences in SYN-IR as a function of age or cognitive status could be attributable to interexperimental variability in immunohistochemical processing. Other aspects of immunohistochemical staining followed previous descriptions (Gazzaley et al., 1996). Briefly, free-floating sections were washed in PBS at room temperature $(3 \times 10 \mathrm{~min})$ and incubated for $48 \mathrm{hr}$ at $4^{\circ} \mathrm{C}$ with antibodies to either SYN or MAP2 diluted in PBS. After being rinsed $(3 \times 10 \mathrm{~min}$ in PBS), the sections were incubated for $1.5 \mathrm{hr}$ with a fluorescein-conjugated antimouse $\operatorname{IgG}$ heavy and light chain secondary antibody (Vector Laboratories, Burlingame, CA). After a final rinse $(3 \times 10 \mathrm{~min}$ in PBS) the sections were mounted onto gelatin-subbed slides and left to dry overnight. Slides were coverslipped with Vectashield (Vector Laboratories) to reduce fluorescence quenching and were stored at $4^{\circ} \mathrm{C}$ until analysis.

Quantitative CLSM. Blind-coded immunolabeled sections through the hippocampus were analyzed with a Zeiss LSM 410 inverted confocal microscope (Oberkochen, Germany) with a Plan-Apochromat $63 \times / 1.25$ numerical aperture oil immersion objective. Fluorescein was excited by an argon/krypton laser at $488 \mathrm{~nm}$, attenuated by a neutral density filter to $3.3 \%$ (attenuation setting of 10), and reflected to the tissue with an FT488/568 dichroic mirror. Settings for gain, aperture, contrast, and brightness were optimized initially and held constant throughout the study so that all sections were digitized under the same conditions of illumination. Given that images from different young and aged cohorts were captured over a period of several months, it was important to evaluate whether system sensitivity varied as a function of waning laser strength or other factors. For this purpose, calibration curves were calculated on days 1,30 , and 60 of image capture by using fluorescein-containing polystyrene microspheres $(2.5 \mu \mathrm{m}$; InSpeck Microscope Image Intensity Calibration Kit, Molecular Probes, Eugene, OR). These commercially available microspheres are manufactured to exhibit seven known decreasing levels of fluorescence intensity, expressed as a relative percentage of maximum intensity. Standard curves derived by this approach are shown in Figure 1. The curves were similar in form and displayed comparable maxima, confirming that system sensitivity was stable over the course of the study.

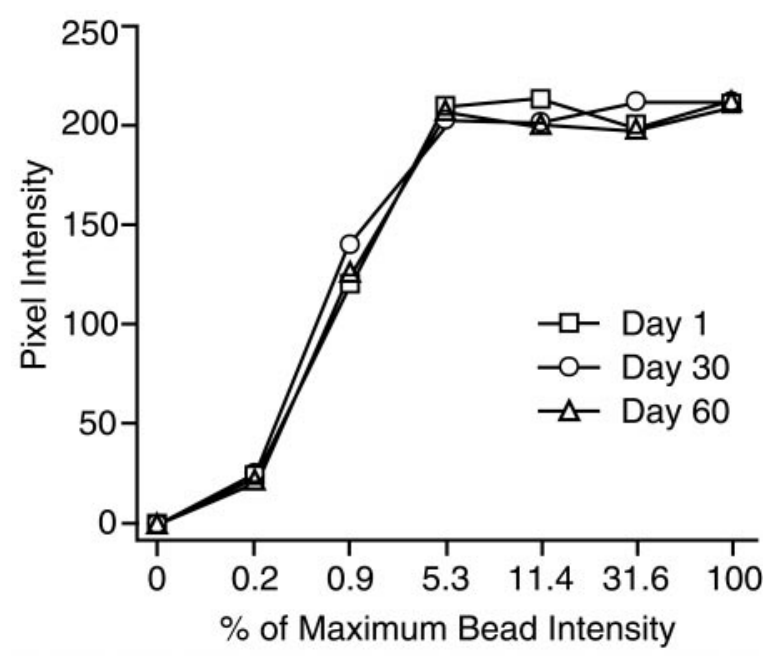

Figure 1. Curves relating measured pixel intensity to relative fluorescence intensity of calibrated microspheres. Images were captured on days 1,30 , and 60 of data collection and used the same CLSM parameters as in the SYN-IR analysis.

Figure 1 also illustrates that the function relating relative fluorescence intensity to measured values was curvilinear under the imaging conditions that were used here. Average fluorescence intensity for SYN-IR in both young and aged brains fell within the rising portion of the curve (see Results), indicating that the CLSM settings were appropriate for detecting potential differences in staining intensity between brains.

Digitized images of SYN-IR and MAP2-IR fluorescence staining were captured from the inner (IML), middle (MML), and outer (OML) portions of the dentate gyrus molecular layer, stratum lucidum (SL), and stratum lacunosum-moleculare (LM) of CA3, and stratum radiatum (SR) and LM of CA1. Using material from a previous study stained by the Timm histochemical method (Rapp et al., 1999), we determined that, in young and aged Long-Evans rats, the IML, MML, and OML comprise 25, 40, and $35 \%$ of the total molecular layer width, respectively. Guided by these observations, we determined laminar boundaries in the present investigation by calculating the total width of the dentate gyrus molecular layer for each of the captured images (i.e., from the granule cell/molecular layer border to the hippocampal fissure or ventricular border) and by applying the corresponding percentages for the IML, MML, and OML. In CA3, SL was distinguished readily as a relatively wide layer of large, bright, punctate immunostaining superficial to the pyramidal cells. This layer tapered to a point at the CA2 border. SR of CA1 was distinguished as the secondary and tertiary dendritic branch points that started $\sim 150 \mu \mathrm{m}$ from the cell body layer and were outlined clearly by the SYN-IR puncta. Images for LM of both CA 3 and CA1 were captured within the zone of the fine most-distal dendrites, $\sim 50 \mu \mathrm{m}$ deep to the hippocampal fissure (Fig. 2).

The overall sampling design was guided by stereological principles, adapted to accommodate the characteristics of the immunohistochemical staining. For each lamina of interest within the dentate gyrus and CA1, one field was sampled every $400 \mu \mathrm{m}$ throughout the transverse axis with a $63 \times / 1.25$ numerical aperture objective. A systematic random selection of sites was ensured by positioning the initial sampling field randomly within the first $400 \mu \mathrm{m}$ interval. The same approach was used in CA3-LM and SL, except that the within-section sampling interval was $100 \mu \mathrm{m}$. Following this design, we sampled an average of $\sim 200$ sites in each hippocampus for a total of $>5000$ samples across the entire study. "Zoomed" images were captured from the center portion of each of the $63 \times$ images, decreasing experimenter bias and potential measurement variability across the display. Each of these individual fields of view, consisting of $512 \times 512 \times$ eight-bit pixel arrays $(400 \times 400 \mu \mathrm{m}$ in the $x-y$ plane), was scanned and digitized, using an electronic zoom factor of 5.0 (increasing the resolution to $0.793 \mu \mathrm{m} /$ pixel and the magnification to $315 \times$ while decreasing the area to $1651 \mu \mathrm{m}^{2}$ ) at a predetermined $z$-axis depth (i.e., distance into the tissue) that remained constant for a given brain. The latter parameter ranged from 3 to $7 \mu \mathrm{m}$ across brains and was selected on the basis of the depth at which staining intensity appeared consistent while avoiding artifacts at the cut surface of the sections. Pixel values, corresponding to the intensity of immunofluorescence staining, were represented on a gray scale of 0 to 255 . Following a procedure outlined by Gazzaley et al. (1996), we applied a photometric offset or "threshold" to each image that distinguished the relatively dim background levels of fluorescence from the more intense punctate staining characteristic of specific SYN-IR (Fig. 2). This approach excludes from analysis the contribution of pixels with gray scale values falling below threshold. The principal measures of interest derived from the captured thresholded images were the average pixel intensity and the cross-sectional area of immunoreactive staining. As discussed later, comparing results across these parameters provided a basis for dissociating two 

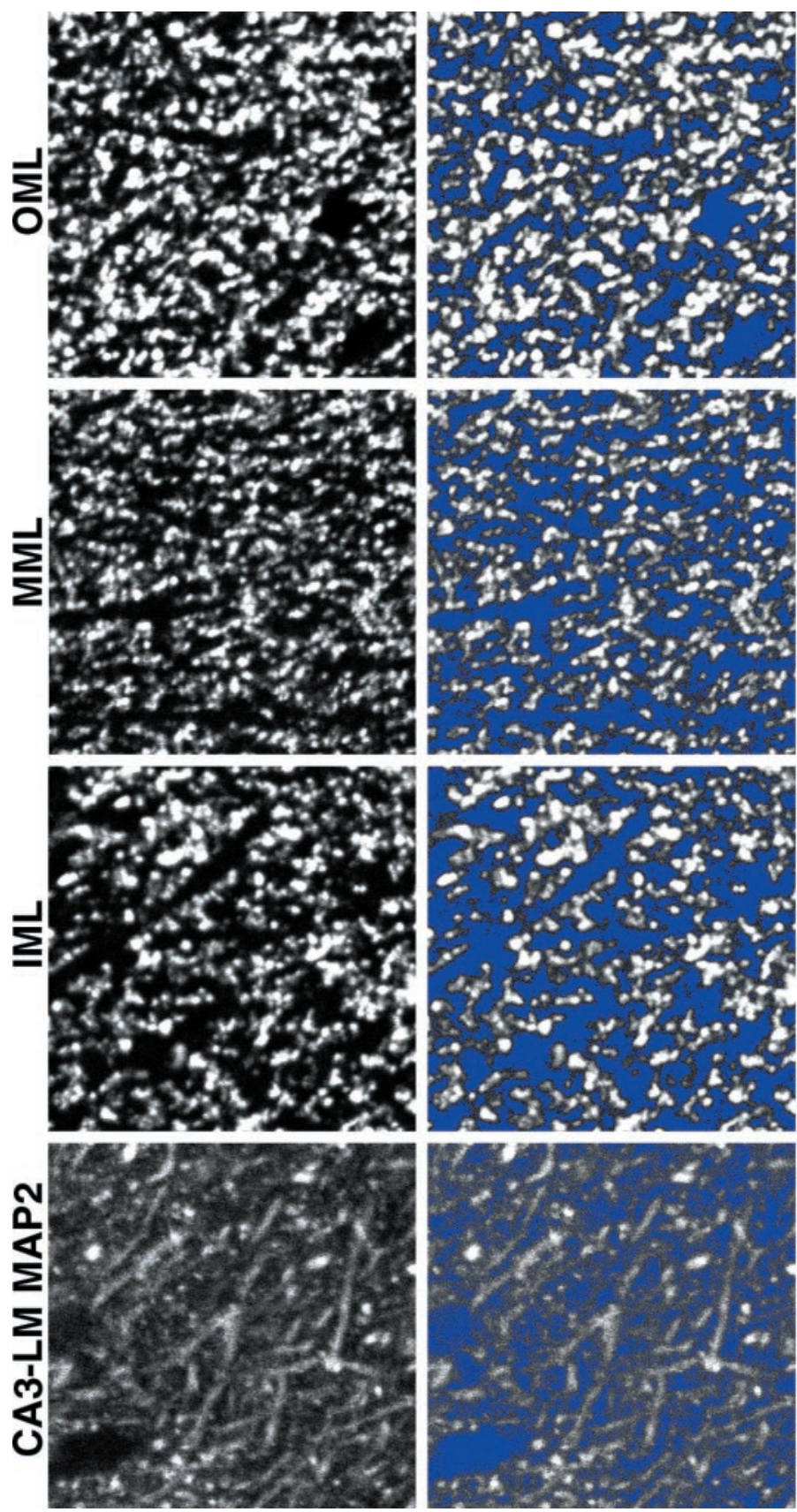
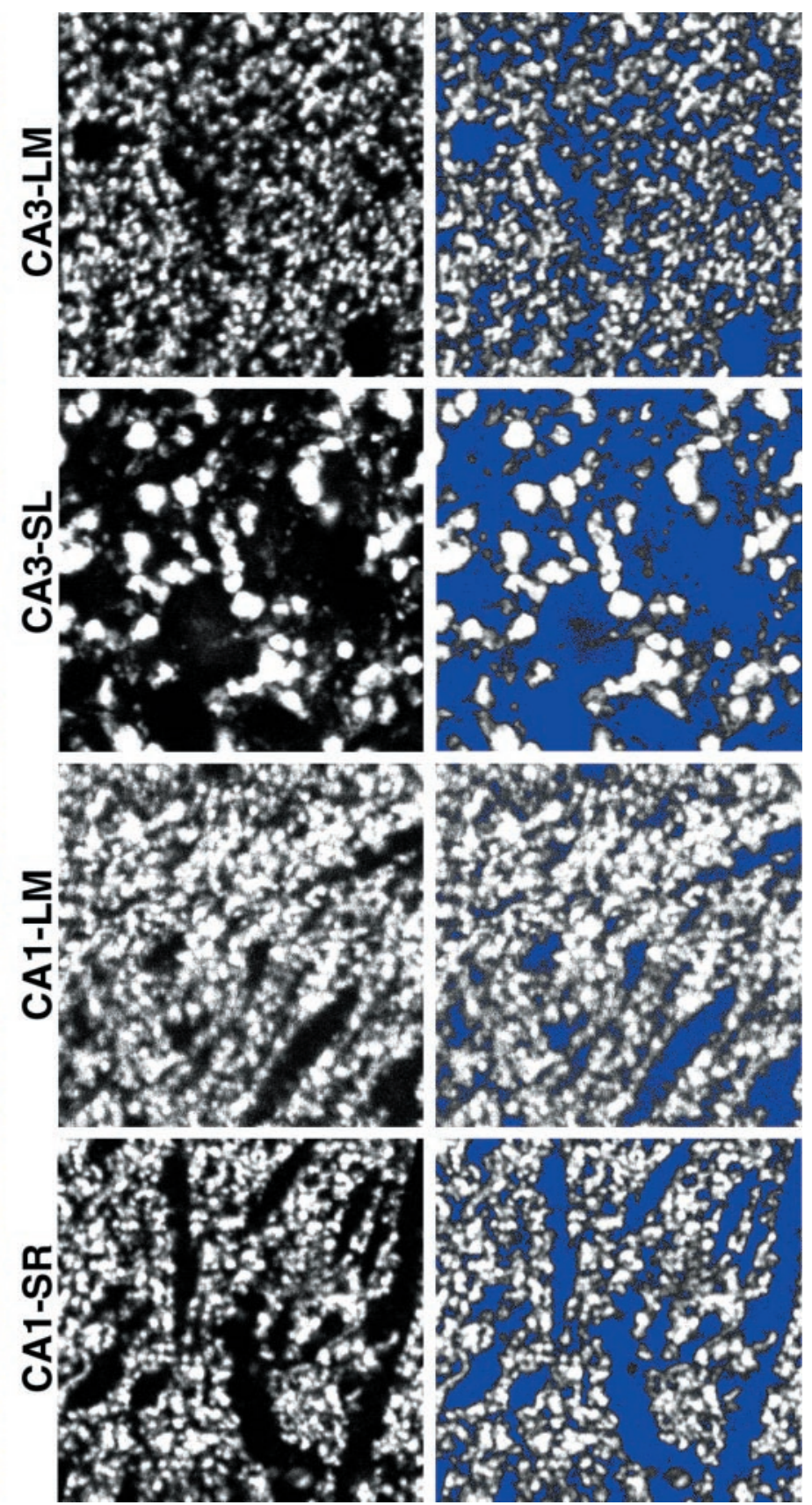

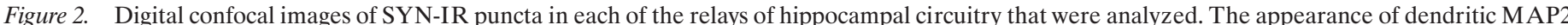

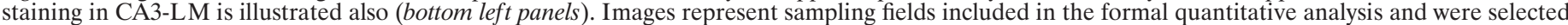

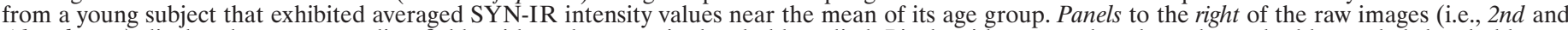

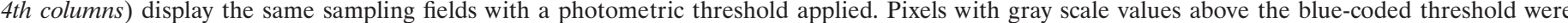

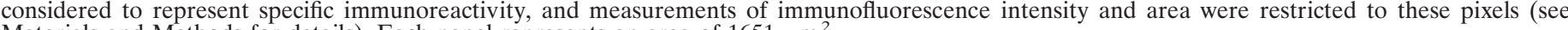
Materials and Methods for details). Each panel represents an area of $1651 \mu \mathrm{m}^{2}$.

potential effects of aging: changes in SYN protein levels versus alterations in the density of presynaptic boutons. Similar procedures were applied to material that was labeled with antibodies against MAP2, providing a window on the overall structural integrity of dendrites in the aged hippocampus.

The blind code was broken after the data collection. Statistical analyses were performed with StatView 5.0 (Abacus Concepts, Berkeley, CA) for the Macintosh platform (Apple Computer, Cupertino, CA). Potential group differences with respect to chronological age and cognitive status were tested by factorial ANOVA and follow-up pair-wise contrasts (Bonferroni test). To examine potential regional selectivity in the effects of aging, we conducted separate tests for each principal hippocampal relay. Adopting a strategy validated in earlier studies that used the same rat model (Baxter and Gallagher, 1996), we used a linear correlation approach (Pearson's $r$ ) to explore the relationship between individual differences in spatial learning and hippocampal SYN-IR. A relatively small number of correlation coefficients was calculated in this analysis (i.e., 19), and, be- cause fewer than one of these would be expected to emerge as significant by chance alone, no correction for multiple comparisons was applied.

\section{RESULTS}

The outcome of behavioral testing was consistent with earlier studies that used identical procedures (Gallagher et al., 1993; Rapp and Gallagher, 1996; Rapp et al., 1999). Briefly, Figure $3 A$ shows the average search error for the young and aged groups during training trials in the hippocampal-dependent place version of the water maze. Performance did not differ as a function of age on the first training trial, but subsequent acquisition was impaired in the aged group. The latter effect was confirmed in a statistical analysis that revealed a main effect of age during training $\left(F_{1,25}=5.18 ; p<0.05\right)$. Learning index scores computed from the interpolated probe trials are shown 


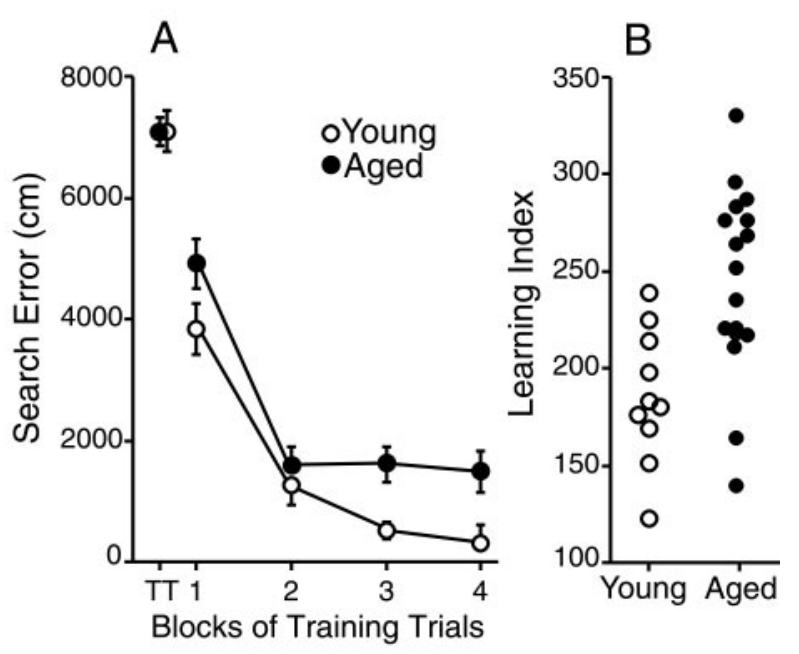

Figure 3. Performance of young $(n=10)$ and aged $(n=17)$ rats in the hippocampal-dependent spatial version of the Morris water maze task. $A$, Average cumulative search error \pm SEM, reflecting the distance of animals from the escape platform throughout their search, over five-trial blocks of training. Performance was nearly identical in young and aged animals on the first training trial (TT) but was impaired in the aged group during subsequent training. $B$, Learning index scores of spatial bias for individual animals derived from three interpolated probe tests in which the platform was unavailable for escape. Because this measure represents average proximity from the platform training location, lower scores are indicative of more accurate searching. Note that learning scores for the aged rats were distributed continuously across a broader range than for young animals.

for each rat in Figure 3B. Lower values of the learning index indicate a more accurate search in the escape platform location. Young rats displayed better learning than the aged subjects $\left(F_{1,25}=11.25 ; p<\right.$ $0.005)$. Note, however, that the range of scores for the aged rats overlapped and exceeded values for the young group; some aged rats performed comparably to young subjects, whereas others scored outside this normative range. Rather than following a strict bimodal distribution, performance in the aged group was distributed continuously across a broader range than for young rats. The results from cue training trials, when rats were allowed to escape to a visible platform, did not differ as a function of age for either escape latency or path length (data not shown).

The overall pattern of SYN-IR in the rat hippocampus, independent of age, was similar to previous descriptions (Fykse et al., 1993; Chen et al., 1995). Bright puncta of SYN-IR were visible in all of the subfields that were examined (see Fig. 2). Staining intensity was higher in CA1-LM than in CA1-SR $\left(F_{1,26}=20.70 ; p<0.0001\right)$ and greater in CA3-SL than in CA3-LM $\left(F_{1,26}=22.39 ; p<0.0001\right)$. Consistent with previous observations (Cabalka et al., 1992; Chen et al., 1995), SYN-IR varied across the radial extent of the dentate gyrus molecular layer $\left(F_{2,52}=106.3 ; p<0.0001\right)$ and was less intense in the MML than in the IML $(p<0.0001)$ or OML $(p<0.0001$; Fig. 4). Labeling in the latter two regions was comparable. These results confirm that the methods used in the present study were sufficiently sensitive to detect subjectively apparent regional differences in the intensity of SYN-IR. By comparison, quantitative ultrastructural data indicate that synaptic density is homogeneous along the distal dendrites of the granule cells and greater than in the IML (Curcio and Hinds, 1983; Desmond and Levy, 1986). Accordingly, available findings are consistent with the idea that SYN-IR can reveal elements of presynaptic circuit organization distinct from synaptic density, presumably related to the size and SYN protein content of immunoreactive boutons (Calhoun et al., 1996)

There were no gross, subjectively apparent differences in the overall quality or general characteristics of SYN labeling in the material from young and aged animals. Statistical comparisons, independent of spatial learning ability, confirmed these observations. Specifically, there was no significant difference in the intensity or cross-sectional area of SYN-IR across the age groups, regardless of whether values were averaged for the entire hippocampus or analyzed separately for each hippocampal subfield (all ANOVA $p$ values $>0.10)$. In a majority of the examined regions, however, average SYN-IR intensity values for the aged group were numerically lower than in the younger cohort (Fig. 4).

Subsequent analyses focused on evaluating the SYN results in relation to variability in the cognitive outcome of aging. On the basis of their performance in the hippocampal-dependent spatial version of the water maze, and adopting a criterion established across many earlier investigations in the same animal model, operationally we defined aged rats with learning index scores $<240$ (i.e., within the range of young controls) as learning-unimpaired. Aged animals with scores outside this normative range were classified as impaired. Previous studies confirm the validity and sensitivity of this strategy for detecting neurobiological alterations that may not be apparent when young and aged subjects are compared on the basis of chronological age alone (for discussion, see Gallagher et al., 1993, 1995; Gallagher and Rapp, 1997). In the present experiments, classifying the aged rats by cognitive status revealed that the numerical decrease in SYN-IR, observed for the aged rats as a group (Fig. 4), was attributable almost entirely to a decline in the subset of aged animals that displayed robust spatial learning deficits (Fig. 5). By comparison, average SYN-IR intensity values for the young and aged-unimpaired groups were virtually identical in the majority of examined regions. This pattern of results was most compelling in CA3-LM, where the SYN staining intensity was reduced by $\sim 28 \%$ in the aged-impaired group relative to either young controls or age-matched animals with preserved spatial learning. Statistical analysis confirmed the presence of a significant group effect for this hippocampal region $\left(F_{2,24}=6.30 ; p<0.01\right)$. Subsequent betweengroup comparisons demonstrated that, whereas SYN-IR intensity values were lower in aged-impaired rats relative to either young $(p<0.01)$ or aged-unimpaired subjects $(p<0.01)$, results for the latter groups were statistically indistinguishable $(p>0.9)$. Although the cross-sectional area of SYN-IR in CA3-LM also differed across groups $\left(F_{2,24}=4.21 ; p<0.05\right)$, this effect was less robust and not statistically reliable in between-group contrasts with appropriate Bonferroni adjustments. Parallel analyses failed to reveal significant group differences in SYN staining intensity or area for any of the remaining hippocampal subfields. Nonetheless, a pattern of results qualitatively similar to CA3-LM was observed in a majority of the examined regions, including the OML and MML (Fig. 5).

The intensity of SYN staining among the aged rats, like their spatial learning scores, was distributed continuously across a broader range than among young subjects. These results raised the possibility that individual differences in spatial learning during aging might be coupled to variability in SYN-IR. This proposal was tested by a linear correlation approach (Pearson's $r$ ). Data from the young and aged groups initially were considered separately, eliminating the risk of detecting associations that simply reflect the shared influence of chronological age on SYN-IR and behavior rather than a meaningful relationship between the latter measures. Lower learning index scores among the aged rats (i.e., better performance) correlated with higher intensity values for SYN-IR selectively in three regions: the dentate gyrus OML $(r=-0.56 ; p=0.02)$, MML $(r=-0.57 ; p=$ $0.02)$, and CA3-LM $(r=-0.63 ; p=0.006)$ (Fig. 6). These results indicate that $\sim 30$ to $40 \%$ of the variance in spatial learning among the aged rats was accounted for by individual differences in SYN-IR in the OML, MML, and CA3-LM. Confirming the regional selectivity of this effect, the correlation was weaker $(r=-0.43)$ and failed to reach standard levels of statistical significance $(p=0.08)$ when SYN-IR values averaged across the entire hippocampus were considered in the analysis.

Parallel analysis of the data from young subjects revealed no statistically reliable correlation between the intensity of SYN labeling and spatial learning, either for the hippocampus as a whole or for any individual subfield (all $p$ values $>0.1$ ). When results for the young and aged rats were pooled, however, the regionally selective inverse correlations that were observed for the aged 

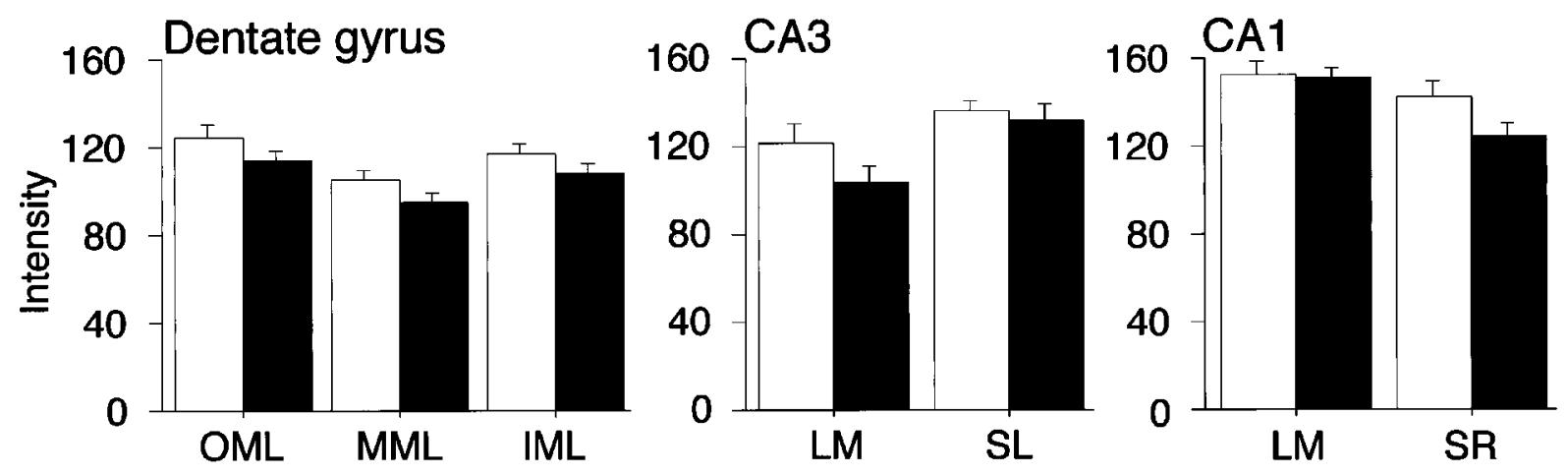

Figure 4. Mean hippocampal SYN-IR intensity \pm SEM for the young (open bars) and aged groups ( filled bars), independent of spatial learning ability. The numerically lower values observed in aged rats were not statistically different from young controls in any region.
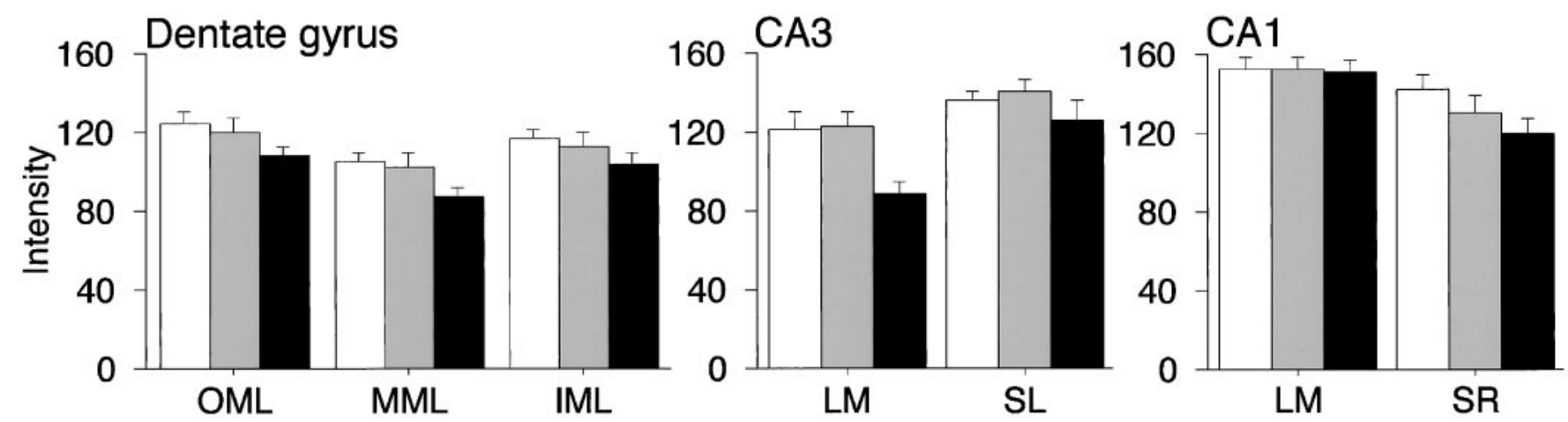

Figure 5. Mean hippocampal SYN-IR intensity \pm SEM in young rats (open bars) and aged animals with (filled bars) or without (shaded bars) spatial learning impairment. Aged animals were classified as impaired $(n=9)$ or unimpaired $(n=8)$ on the basis of their performance in the place version of the water maze (see Fig. $2 B$ and Results for details). Note that average hippocampal SYN-IR intensity values were statistically indistinguishable between aged-unimpaired and young rats in all of the regions that were examined. The subset of aged rats with robust spatial learning deficits, however, displayed significantly lower SYN-IR intensity values in CA3-LM relative to either young animals or age-matched rats with preserved learning.
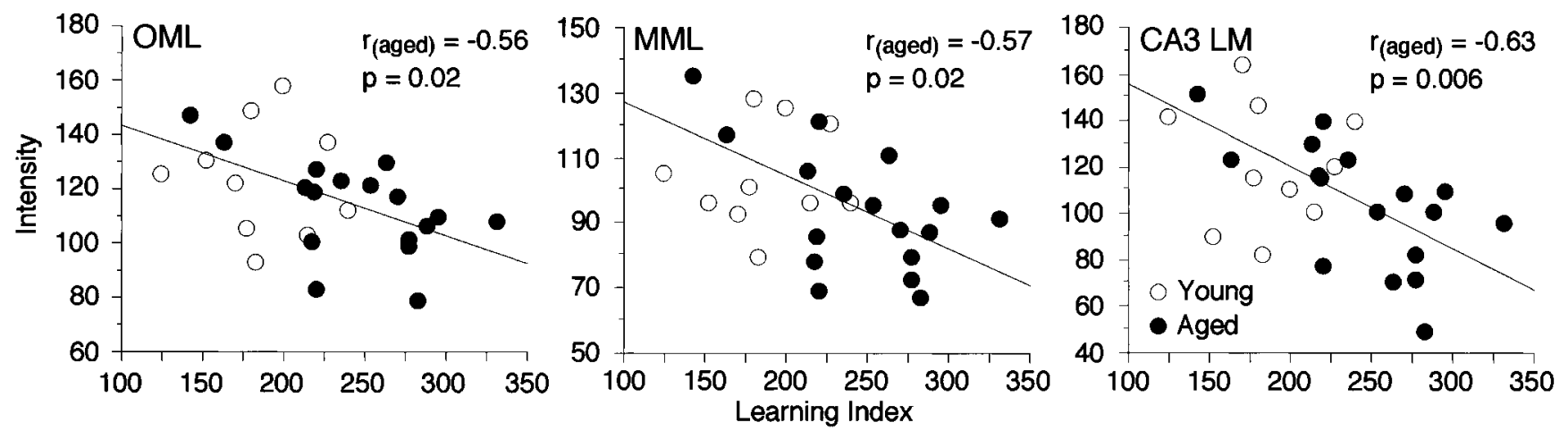

Figure 6. Scatter plots relating individual spatial learning index scores to levels of hippocampal SYN-IR in young and aged rats. Lower learning index scores, indicative of better learning, correlated with higher SYN-IR intensity values selectively in the dentate gyrus OML, MML, and CA3-LM. Regression lines and correlation coefficients refer to the relationship between individual differences in spatial learning and SYN-IR for the aged subjects alone.

subjects alone remained significant (OML: $r=-0.46, p=0.02$; MML: $r=-0.46, p=0.01$; CA3-LM: $r=-0.54, p=0.003)$. This pattern of results suggests that SYN-IR in specific components of hippocampal circuitry may be coupled to spatial learning across the full range of performance capacities observed in young and aged rats. By this account the failure to detect a corresponding association for the young group alone might reflect the limited statistical power provided by considering only 10 subjects in the analysis. Alternatively, hippocampal SYN-IR may be coupled less tightly to learning in young animals, emerging selectively when the effects of aging on relevant circuitry exceed some critical threshold.

In contrast to the intensity of SYN-IR, the cross-sectional area of immunostaining failed to correlate with the learning index scores in any hippocampal regions for either the young or aged animals (no $r$ values better than -0.30 ; all $p$ values $>0.1$ ). Thus, individual variability in the numerical density of presynaptic boutons, which would influence the cross-sectional area of SYN staining directly, is unlikely to account for the observed coupling between spatial learning ability and the intensity of immunoreactivity.

Finally, alternate histological sections stained with antibodies against the cytoskeletal protein MAP2 were examined to evaluate the possibility that the observed changes in SYN-IR were associated with generalized dendritic deterioration in the aged hippocampus. There were no significant age-related differences in either the intensity or area of MAP2-IR in any brain region that was examined (all $p$ values $>0.1$ ). In addition, MAP2 staining was comparable among the young, aged-unimpaired, and impaired groups (all $p$ values $>0.1)$. Negative results also were obtained in analyses testing 
for potential correlations between the spatial learning scores and the MAP2 measures (no $r$ values better than -0.30 or 0.30 ; all $p$ values $>0.1)$.

\section{DISCUSSION}

The present investigation substantially advances current evidence, providing the first demonstration that variability in the cognitive outcome of normal aging is coupled to circuit-specific alterations in the organization of hippocampal connectivity. Comparisons on the basis of chronological age alone failed to reveal any reliable difference in SYN-IR, either for the hippocampus as a whole or in individual relays of hippocampal circuitry. In contrast, aged rats with robust spatial learning deficits displayed significant reductions in the intensity of SYN-IR in CA3-LM relative to both younger animals and age-matched subjects with preserved learning. Individual variability in hippocampal learning among the aged rats correlated with SYN immunolabeling selectively in three areas: the OML, MML, and CA3-LM. This regional specificity, together with the observation that SYN-IR was entirely normal in aged rats without spatial impairment, argues against a general, nonspecific change in the immunohistochemical staining characteristics of the aged brain. Given that all of the affected regions receive a prominent projection from layer II of the entorhinal cortex, the results instead suggest that cognitive aging is linked to a decline in the integrity of multimodal associational input that these cells relay to the hippocampus. The absence of a corresponding alteration in the cross-sectional area of SYN staining is also informative, implying that presynaptic alterations coupled to age-related learning deficits include a decrease in hippocampal SYN protein levels that is unaccounted for by changes in terminal density.

The findings reported here help to reconcile available data on the status of connectivity in the aged hippocampus. In a recent experiment that used the same animal model, Nicolle et al. (1999b) found no differences related to age or cognitive status in the amount of three presynaptic proteins, including SYN, measured by Western blotting in whole homogenized hippocampi. Our results are compatible with those findings in that the overall intensity of SYN-IR, averaged across the principal relays of hippocampal circuitry, failed to differ as a function of chronological age or cognitive status. Studies incorporating greater anatomical resolution, however, suggest that there is substantial regional selectivity in the effects of aging on hippocampal circuitry, particularly in relation to cognitive decline (Geinisman et al., 1986; deToledo-Morrell et al., 1988a,b; Rapp et al., 1999). Consistent with this conclusion, agerelated alterations in SYN-IR in the present experiment were apparent only among aged rats with pronounced spatial learning impairment. In addition, correlations between SYN intensity and behavior were selective for subfields of the hippocampus that receive direct cortical input (see below). In the absence of discrete regional analysis and assessment of capacities supported by the hippocampus, this pattern of results would be obscured.

Although quantitative electron microscopy remains the only definitive means of determining synapse number, SYN-IR provides a sensitive marker of the synapse loss that accompanies neurodegenerative disease (Goto and Hirano, 1990; Cabalka et al., 1992; Masliah et al., 1993, 1994, 1995; Heinonen et al., 1995). In the present experiments the overall density of presynaptic boutons, measured as the cross-sectional area of SYN-IR, was not reliably different as a function of chronological age or cognitive status. Chen et al. (1995) reported essentially the same result, suggesting that any effect of normal aging on presynaptic terminal number in the hippocampus is sufficiently subtle to elude detection by the approach used in these studies. Our additional findings, however, demonstrate marked effects on the intensity of labeling for this presynaptic protein. The interpretation of these results is informed by the observation that age-related alterations in SYN-IR were apparent selectively among aged rats with documented deficits in spatial learning and only in certain relays of hippocampal circuitry. In addition, no change with chronological age or cognitive status was detected in a parallel analysis of labeling for the cytoskeletal protein
MAP2. A distinctly different pattern of results also was obtained when immunoreactivity for the NR1 subunit of the NMDA receptor was quantified in alternate histological sections from the same animals (M. Adams, T. Smith, M. Gallagher, P. Rapp, and J. Morrison, unpublished data). Differences in antibody penetration or other nonspecific alterations in the aged brain cannot account for this regional, behavioral, and protein specificity. Taken together, the results instead suggest that cognitive aging is linked to circuitspecific alterations in presynaptic elements of hippocampal connectivity (e.g., vesicle number, transmitter packaging, etc.), independent of effects on the overall density of presynaptic boutons.

Synaptophysin and other synaptic vesicle proteins have been implicated in mechanisms of cellular plasticity that are thought to underlie learning (Lynch et al., 1994; Mullany and Lynch, 1998; Janz et al., 1999). Viewed in the context of the overall circuit organization of the hippocampal formation (for review, see Amaral and Witter, 1995), this evidence provides a valuable framework for evaluating the functional implications of the present results. Inputs from widespread regions of the neocortex converge in the entorhinal cortex and are relayed to the distal dendrites of granule cells in middle and outer portions of the dentate gyrus molecular layer. Output from the granule cells is directed to CA3 pyramidal neurons that, in turn, project to the CA1 field. Superimposed on this classic trisynaptic circuitry, the entorhinal cortex also projects directly to hippocampal pyramidal cells. These pathways follow a laminar organization such that input to CA3-LM arises principally from the same layer II neurons that innervate the dentate gyrus. In contrast, entorhinal cortex projections to CA1-LM originate exclusively in layer III. Thus, the present results indicate that individual differences in the cognitive outcome of aging are coupled to SYN-IR selectively in regions of the hippocampus that receive direct input from layer II of the entorhinal cortex (i.e., the OML, MML, and CA3-LM). Terminal fields targeted by intrinsic projections (e.g., the IML, CA3-SL, and CA1-SR) or layer III neurons of the entorhinal cortex (CA1-LM) are relatively unaffected. This pattern of results highlights that the morphometric effects of aging are remarkably selective, preferentially affecting circuits that convey cortically derived information that is critical for hippocampal learning.

Recent findings substantially constrain the basis of age-related change in hippocampal circuitry. Although it might be supposed that the regional selectivity demonstrated in the current investigation results from a degeneration of layer II neurons in the entorhinal cortex, recent stereological studies indicate that neuron number is preserved throughout many fields of hippocampal formation during normal aging (West, 1993; Rapp, 1995; Peters et al., 1996; Rapp and Gallagher, 1996; Rasmussen et al., 1996; Calhoun et al., 1998). In addition, sparing has been noted in several reports that specifically examined neuron number in layer II of entorhinal cortex (Rapp, 1995; Gomez-Isla et al., 1996; Gazzaley et al., 1997; Merrill et al., 2000). Thus, although a definitive answer awaits parallel analysis in the rat model used here, there is little empirical support for the idea that alterations in the circuit organization of the aged hippocampus are a secondary consequence of neuron loss in the entorhinal cortex. The present results also appear unrelated to gross structural deterioration in the dendrites of hippocampal cells that receive afferents from the entorhinal cortex. In addition to evidence for preserved or increased dendritic elaboration until very late in life (for review, see Coleman and Flood, 1987; Turner et al., 1998), our results failed to reveal any change with age in the area or intensity of staining for the cytoskeletal protein MAP2. Taken together, the findings point to changes in protein conformation, trafficking, or other biochemical alterations as more plausible candidates underlying the observed effects of aging on SYN-IR.

The circuit-specific effects documented in this study are positioned to influence the computational function of the hippocampus at multiple levels of processing. Specifically, our results imply that the fidelity of entorhinal input to the dentate gyrus declines in a subpopulation of aged rats. The outcome of processing this degraded information is relayed to CA3 where, modulated by disrupted direct inputs from layer II of the entorhinal cortex, additional computa- 
tional errors may accrue. Recent electrophysiological studies confirm that the encoding properties of hippocampal pyramidal neurons are altered substantially in aged rats with spatial learning deficits (Barnes et al., 1997; Shen et al., 1997; Tanila et al., 1997a,b). Of particular interest, location-specific firing of hippocampal neurons in these animals appears to be controlled by a more limited scope of available environmental cues than in young rats or age-matched subjects with preserved spatial learning. Taken together with the present findings, it is tempting to speculate that this impoverished encoding results from age-related deterioration in the integrity of multimodal sensory input to the hippocampus from the entorhinal cortex. Computational modeling of hippocampal function, mimicking the modest regionally selective alterations reported here, could provide a useful strategy for testing this account.

\section{REFERENCES}

Amaral DG, Witter MP (1995) Hippocampal formation. In: The rat nervous system, 2nd Ed (Paxinos G, ed), pp 443-493. San Diego: Academic.

Barnes CA (1999) Do synaptic markers provide a window on synaptic effectiveness in the aged hippocampus? Neurobiol Aging 20:349-351.

Barnes CA, Suster MS, Shen J, McNaughton BL (1997) Multistability of cognitive maps in the hippocampus of old rats. Nature 388:272-275.

Baxter MG, Gallagher M (1996) Neurobiological substrates of behavioral decline: models and data analytic strategies for individual differences in aging. Neurobiol Aging 17:491-495.

Bernhardt R, Matus A (1984) Light and electron microscopic studies of the distribution of microtubule-associated protein 2 in rat brain: a difference between dendritic and axonal cytoskeletons. J Comp Neurol 226:203-221.

Cabalka LM, Hyman BT, Goodlett CR, Ritchie TC, Van Hoesen G (1992) Alteration in the pattern of nerve terminal protein immunoreactivity in the perforant pathway in Alzheimer's disease and in rats after entorhinal lesions. Neurobiol Aging 13:283-291.

Calhoun ME, Jucker M, Martin LJ, Thinakaran G, Price DL, Mouton PR (1996) Comparative evaluation of synaptophysin-based methods for quantification of synapses. J Neurocytol 25:821-828.

Calhoun ME, Kurth D, Phinney AL, Long JM, Hengemihle J, Mouton PR, Ingram DK, Jucker M (1998) Hippocampal neuron and synaptophysinpositive bouton number in aging. Neurobiol Aging 19:599-606.

Chen KS, Masliah E, Mallory M, Gage FH (1995) Synaptic loss in cognitively impaired aged rats is ameliorated by chronic human nerve growth factor infusion. Neuroscience 68:19-27.

Coleman PD, Flood DG (1987) Neuron numbers and dendritic extent in normal aging and Alzheimer's disease. Neurobiol Aging 8:521-545.

Curcio CA, Hinds JW (1983) Stability of synaptic density and spine volume in dentate gyrus of aged rats. Neurobiol Aging 4:77-87.

Desmond NL, Levy WB (1986) Changes in the numerical density of synaptic contacts with long-term potentiation in the hippocampal dentate gyrus. J Comp Neurol 253:466-475.

deToledo-Morrell L, Geinisman Y, Morrell F (1988a) Individual differences in hippocampal synaptic plasticity as a function of aging: behavioral, electrophysiological, and morphological evidence. In: Neural plasticity: a lifespan approach (Petit TL, Ivy GO), pp 283-328. New York: Liss.

deToledo-Morrell L, Geinisman Y, Morrell F (1988b) Age-dependent alterations in hippocampal synaptic plasticity: relation to memory disorders. Neurobiol Aging 9:581-590.

Fykse EM, Takei K, Walch-Solimena C, Geppert M, Jahn R, De Camilli P, Südhof T (1993) Relative properties and localizations of synaptic vesicle protein isoforms: the case of the synaptophysins. J Neurosci 13:4997-5007.

Gallagher M, Rapp PR (1997) The use of animal models to study the effects of aging on cognition. Annu Rev Psychol 48:339-370.

Gallagher M, Burwell R, Burchinal M (1993) Severity of spatial learning impairment in aging: development of a learning index for performance in the Morris water maze. Behav Neurosci 107:618-626.

Gallagher M, Nagahara AH, Burwell RD (1995) Cognition and hippocampal systems in aging: animal models. In: Brain and memory: modulation and mediation of neuroplasticity (McGaugh JL, Weinberger N, Lynch G, eds), pp 103-126. New York: Oxford UP.

Gallagher M, Landfield PW, McEwen B, Meaney MJ, Rapp PR, Sapolsky R, West MJ (1996) Hippocampal neurodegeneration in aging [letter]. Science 274:484-485.

Gazzaley AH, Siegel SJ, Kordower JH, Mufson EF, Morrison JH (1996) Circuit-specific alterations of $N$-methyl-D-aspartate receptor subunit 1 in the dentate gyrus of aged monkeys. Proc Natl Acad Sci USA 93:3121-3125.

Gazzaley AH, Thakker MM, Hof PR, Morrison JH (1997) Preserved number of entorhinal cortex layer II neurons in aged macaque monkeys. Neurobiol Aging 18:549-553.

Geinisman Y (1999) Age-related decline in memory function: is it associated with a loss of synapses? Neurobiol Aging 20:353-356.

Geinisman Y, deToledo-Morrell L, Morrell F (1986) Aged rats need a preserved complement of perforated axospinous synapses per hippocampal neuron to maintain good spatial memory. Brain Res 398:266-275.

Geinisman Y, deToledo-Morrell L, Morrell F, Heller RE (1995) Hippocampal markers of aged-related memory dysfunction: behavioral, electrophysiological, and morphological perspectives. Prog Neurobiol 45:223-252.

Gomez-Isla T, Price JL, McKeel Jr DW, Morris JC, Growdon JH, Hyman BT (1996) Profound loss of layer II entorhinal cortex neurons occurs in very mild Alzheimer's disease. J Neurosci 16:4491-4500.

Good MJ, Hage WJ, Mummery CL, De Laat SW, Boonstra J (1992) Localization and quantification of epidermal growth factor receptors on single cells by confocal laser scanning microscopy. J Histochem Cytochem 40:1353-1361.

Goto S, Hirano A (1990) Synaptophysin expression in the striatum in Huntington's disease. Acta Neuropathol (Berl) 80:88-91.

Heinonen O, Soininen H, Sorvari H, Kosunen O, Paljarvi L, Koivisto E, Riekkinen Sr PJ (1995) Loss of synaptophysin-like immunoreactivity in the hippocampal formation is an early phenomenon in Alzheimer's disease. Neuroscience 62:375-384.

Janz R, Südhof TC, Hammer RE, Unni V, Siegelbaum SA, Bolshakov VY (1999). Essential roles in synaptic plasticity for synaptogyrin I and synaptophysin I. Neuron 24:687-700.

Lynch MA, Voss KL, Rodriguez J, Bliss TV (1994) Increase in synaptic vesicle proteins accompanies long-term potentiation in the dentate gyrus. Neuroscience 60:1-5.

Masliah E, Mallory M, Hansen L, DeTeresa R, Terry RD (1993) Quantitative synaptic alterations in the human neocortex during normal aging. Neurology 43:192-197.

Masliah E, Mallory M, Hansen L, DeTeresa R, Alford M, Terry R (1994) Synaptic and neuritic alterations during the progression of Alzheimer's disease. Neurosci Lett 174:67-72.

Masliah E, Mallory M, Ge N, Alford M, Veinbergs I, Roses AD (1995) Neurodegeneration in the central nervous system of ApoE-deficient mice. Exp Neurol 136:107-122.

Merrill DA, Roberts JA, Tuszynski MH (2000) Conservation of neuron number and size in entorhinal cortex layers II, III, and V/VI of aged primates. J Comp Neurol 422:396-401.

Morris RGM, Garrud P, Rawlins NJP, O'Keefe JO (1982) Place navigation impaired in rats with hippocampal lesions. Nature 297:681-683.

Morrison JH, Hof PR (1997) Life and death of neurons in the aging brain. Science 278:412-419.

Mullany PM, Lynch MA (1998) Evidence for a role for synaptophysin in expression of long-term potentiation in rat dentate gyrus. NeuroReport 9:2489-2494.

Nicolle MM, Gallagher M, McKinney M (1999a) Analyses of hippocampal circuitry in aging. Neurobiol Aging 20:359-360.

Nicolle MM, Gallagher M, McKinney M (1999b) No loss of synaptic proteins in the hippocampus of aged, behaviorally-impaired rats. Neurobiol Aging 20:343-348.

Peters A, Rosene DL, Moss MB, Kemper TL, Abraham CR, Tigges J, Albert MS (1996) Neurobiological basis of age-related cognitive decline in the rhesus monkey. J Neuropathol Exp Neurol 55:861-874.

Rapp PR (1995) Cognitive neuroscience perspectives on aging in nonhuman primates. In: Emotion, memory and behavior (Nakajima T, Ono T, eds), pp 197-211. Tokyo: Japan Scientific Societies.

Rapp PR, Gallagher M (1996) Preserved neuron number in the hippocampus of aged rats with spatial learning deficits. Proc Natl Acad Sci USA 93:9926-9930.

Rapp PR, Stack EC, Gallagher M (1999) Morphometric studies of the aged hippocampus. I. Volumetric analysis in behaviorally characterized rats. J Comp Neurol 403:459-470.

Rasmussen T, Schliemann T, Sorensen JC, Zimmer J, West M (1996) Memory impaired aged rats: no loss of principal hippocampal and subicular neurons. Neurobiol Aging 17:143-147.

Rosene DL (1993) Comparing age-related changes in the basal forebrain and hippocampus of the rhesus monkey. Neurobiol Aging 14:669-670.

Shen J, Barnes CA, McNaughton BL, Skaggs WE, Weaver KL (1997) The effect of aging on experience-dependent plasticity of hippocampal place cells. J Neurosci 17:6769-6782.

Smith TD, Calhoun ME, Rapp PR (1999) Circuit and morphological specificity of synaptic change in the aged hippocampal formation. Neurobiol Aging 20:357-358.

Tanila H, Shapiro M, Gallagher M, Eichenbaum H (1997a) Brain aging: changes in the nature of information coding by the hippocampus. J Neurosci 17:5155-5166.

Tanila H, Sipila P, Shapiro M, Eichenbaum H (1997b) Brain aging: impaired coding of novel environmental cues. J Neurosci 17:5167-5174.

Turner DA, Buhl EH, Hailer NP, Nitsch R (1998) Morphological features of the entorhinal-hippocampal connection. Prog Neurobiol 55:537-562.

West MJ (1993) Regionally specific loss of neurons in the aging human hippocampus. Neurobiol Aging 14:287-293.

Wiedenmann B, Franke WW (1985) Identification and localization of synaptophysin, an integral membrane glycoprotein of $M_{\mathrm{r}} 38,000$ characteristic of presynaptic vesicles. Cell 41:1017-1028. 